\title{
Comparing the effectiveness between mid urethral retro pubic Tvt and transobturator tot or Tvt-o in mixed pattern urinary incontinence with stress incontinence predominating
}

\section{Introduction and objectives}

We compared the effectiveness of mid urethral Retro pubic TVT and Transobturator TVT-O or TOT performed in single centre in female patients with mixed pattern urinary incontinence with stress Incontinence predominating. .

\section{Materials and methods}

A detailed study of the operating log and the patients follow-up notes was performed to identify the patients treated with mid urethral tapes for mixed pattern urinary incontinence with stress in continence predominating. From March 2006 till March 2011, 74 females patients were identified with symptoms of mixed pattern urinary incontinence with stress incontinence predominating. Thirty eight out of $74(51.35 \%)$ underwent retro pubic TVT insertion, 28/74(37.83\%) underwent Transobturator (TVT-O/TOT tapes), 9.45\% (7/74) mini arc and 1 TVT Secure. Fifteen out of $74(20.27 \%)$ patients had no prior urodynamic study. Only $3 / 59$ (5.08\%) patients who underwent urodynamic study showed mild bladder over activity and rest had sensory urgency. Of these 3, 2 underwent Transobturator tape and 1 mini arc. .

\section{Results}

In the TVT (Retro pubic) group 60.52\% (23/38) patients were fully continent while $8 / 38(21.05 \%)$ had partial improvement even with anti cholinergics. Out of these 23 patients $10(43.47 \%)$ needed anti cholinergics while 5/23 $(21.73 \%)$ needed Botulinum toxin in addition. While in the Transobturator group 24/28 (85.71\%) patients were completely continent without complications and only $7 / 24$ $(29.16 \%)$ needed anti cholinergics and $1(4.1 \%)$ needed Botulinum toxin. One out of $28(3.57 \%)$ had partial improvement. $3.57 \%(1 / 28)$ had late failure after initial complete response for 3years. Two out of 3 patients which showed bladder over activity on urodynamics study and underwent Transobturator tape had complete response without any additional treatment. Six out of $38(15.78 \%)$ patients in TVT group presented post op with dysfunctional voiding compared to none in TOT/TVT-O group. Out of these, 2 needed tape division and 1 needed chronic SIC and 1 pt needed early SIC only. In TVT arm 3/38 (7.89\%) had AUR all had chronic diff voiding 2 on SIC and 1 Partial response while none in TOT group. In TOT/TVT-O group $3.57 \%(1 / 28)$ Perennial pain needing tape division and $3.57 \%(1 / 28)$ short-term leg pain was seen but not in TVT group. ${ }^{1-3}$
Volume 2 Issue 4 - 2015

\author{
Ashwini Gaur, Peter Guy \\ University Hospital of North Tees, United Kingdom
}

Correspondence: Ashwini Gaur, Locum Consultant Urological Surgeon, University Hospital of North Tees, Leicester, Leicestershire, United Kingdom,

Email ashwinigaur@hotmail.com

Received: May 18, 2015 | Published: May 22, 2015

\section{Conclusion}

Transobturator tapes are more efficacious and should be considered as first choice in such patients.

\section{Acknowledgements}

None.

\section{Conflict of interest}

The author declares no conflict of interest.

\section{References}

1. Tahseen S, Reid P. Effect of transobturator tape on overactive bladder symptoms and urge urinary incontinence in women with mixed urinary incontinence. Obstet Gynecol. 2009;113(3):617-623.

2. Abdel-Fattah M, Hopper LR, Mostafa A. Evaluation of transobturator tension-free vaginal tapes in the surgical management of mixed urinary incontinence:3-year outcomes of a randomized controlled trial. J Urol. 2014;191(1):114-119.

3. Abdel-fattah M, Ramsay I, Pringle S, et al. Evaluation of transobturator tapes (E-TOT) study: randomised prospective single-blinded study comparing inside-out vs. outside-in transobturator tapes in management of urodynamic stress incontinence: short term outcomes. Eur J Obstet Gynecol Reprod Biol. 2010;149(1):106-111. 